\title{
Robotic guidance induces long-lasting changes in the movement pattern of a novel sport-specific motor task
}

\author{
Jakob Kümmel*, Andreas Kramer, Markus Gruber \\ Sensorimotor Performance Lab, Department of Sport Science, University of Konstanz, Germany
}

Keywords:

Haptic guidance

Endpoint-controlled robot

Golf swing

Motor learning

\begin{abstract}
A B S T R A C T
Facilitating the learning or relearning of motor tasks is one of the main goals of coaches, teachers and therapists. One promising way to achieve this goal is guiding the learner through the correct movement trajectory with the help of a robotic device. The aim of this study was to investigate if haptic guidance can induce long lasting changes in the movement pattern of a complex sport specific motor task. For this purpose, 31 subjects were assigned to one of three groups: EA (early angle, $n$ 10), LA (late angle, $n$ 11) and CON (control, $n$ 10). EA and LA successfully completed five training sessions, which consisted of 50 robot guided golf swings and 10 free swings each, whereas CON had no training. The EA group was guided through the movement with the wrist being bent early during backswing, whereas in the LA group it was bent late. The participants of EA and LA were not told about this difference in the movement patterns. To assess if the robot guided training was successful in shaping the movement pattern, the timing of the wrist bending during the backswing in free swings was measured before (PRE), one day after (POST), and 7 days after (FUP) the five training sessions. The ANOVA (time $\times$ group $\times$ angle) showed that during POST and FUP, the participants of the EA group bent their wrist significantly earlier during the backswing than the other groups. Post hoc analyses revealed that this interaction effect was mainly due to the differences in the wrist angle progression during the first $5^{\circ}$ of the backswing. The robot guided training was successful in shaping the movement pattern, and these changes persisted even after 7 days
\end{abstract}

\footnotetext{
* Corresponding author. Address: FG Sportwissenschaft, Universität Konstanz, 78457 Konstanz, Germany. Tel.: +49 (0) 7531 884213.
}

E-mail address: jakob.kuemmel@uni-konstanz.de (J. Kümmel). 
without further practice. This might have implications for the learning of complex motor tasks in general, as haptic guidance might quickly provide the beginner with an internal model of the correct movement pattern without having to direct the learner's attention towards the key points of the correct movement pattern.

\section{Introduction}

When learning a new motor skill, learners often require a lot of time to develop a movement pat tern that enables them to achieve the movement goal and that is reproducible and stable after a longer period without practice. This is especially true for more complex motor skills, as learning a complex motor skill requires a high amount of information processing capacity (Wulf \& Shea, 2002), which often prevents beginners from concentrating on all of the important elements of the movement. This can cause large initial errors (Wulf \& Shea, 2002), which then can derogate the learning process itself (Sanger, 2004).

A potential solution for this problem is guidance or physical assistance, which might reduce the attentional demands by providing a "perceptual motor workspace" for the learner (Newell, 1991; Wulf \& Shea, 2002; Wulf, Shea, \& Whitacre, 1998). Several studies have examined the beneficial effects of haptic guidance in different areas of motor learning (for review see Sigrist, Rauter, Riener, \& Wolf, 2013). One experimental paradigm that has been used to investigate the effects of haptic guidance in sensorimotor learning is position control during trajectory learning. A haptic interface guides the lear ner along predefined multidimensional trajectories in order to provide a correct movement pattern and to prevent errors during the learning process (Reinkensmeyer \& Patton, 2009). Furthermore, haptic guidance has the capacity to deliver more movement repetitions than conventional training protocols (Huang \& Krakauer, 2009). Hence it has also been applied as a new approach in neuroreha bilitation. However, studies with stroke patients yielded controversial results (Kwakkel, Kollen, \& Krebs, 2008; Prange, Jannink, Groothuis Oudshoorn, Hermens, \& Ijzerman, 2006). This might be due to the fact that studies in this field are often underpowered; hence one might not distinguish the actual treatment effect in a heterogeneous stroke population (Kwakkel et al., 2008). In addition there was a lack of consensus about appropriate outcome measures in order to quantify motor re learning without any compensation strategies (for detailed information see Sivan, O'Connor, Makower, Levesley, \& Bhakta, 2011). Future studies should consider an intensity and duration matched training design to verify improvements that are related to the treatment modalities and not to a higher dose response under robotic rehabilitation condition (for review see Norouzi Gheidari, Archambault, \& Fung, 2012). To sum up the findings of these meta analyses, it is not yet clear whether haptic guidance in rehabilitation is superior to conventional rehabilitation treatments or just provides an alternative treatment possibility (Sigrist et al., 2013).

With healthy subjects, there are only few studies focusing on the use of haptic guidance to help position control during motor skill learning (Feygin, Keehner, \& Tendick, 2002; Liu, Cramer, \& Reinkensmeyer, 2006). In the study by Feygin et al. (2002), the participants learned to track a novel arm movement in three different ways. The first group trained by solely observing the end effector moving along its trajectory. In the second group, the hand of the subject was attached to the end effec tor while it moved along the trajectory with the line of sight to the apparatus being blocked. The third group experienced the same guidance as the second group but additionally was able to see the move ment. The training consisted of 30 movement demonstrations for each group with a recall phase after every second trajectory presentation, during which the subjects had to reproduce the learned move ment trajectory. The authors demonstrated that haptic guidance increased position and especially timing accuracy of the learned movement. 
A similar experiment was carried out by Liu et al. (2006). In their study, the participants had to learn an arm movement via either haptic guidance including vision, or visual training alone. After every seventh trajectory presentation, the tracing error was measured during a recall phase, which consisted of seven free trials. The tracing error decreased for both groups to a similar extent indicating a similar learning effect. These results are in line with the findings of Feygin et al. (2002) and demon strate a high potential for the use of haptic guidance in teaching generic movement trajectories.

However, it is not clear if these results can be transferred to sport specific movements, which are often much more complex than the movement patterns used in the experiments described above. In comparison to the movements examined in previous studies, sport specific movements such as a golf swing, a tennis stroke or a soccer kick involve multi joint whole body movements that require precise coordination of the moving limbs in space and time, together with the necessary postural adjustments.

In the present study, we aimed to assess whether haptic guidance using an endpoint controlled robot can induce long lasting changes in the movement pattern of a novel sport specific motor task.

\section{Methods}

\subsection{Subjects}

At the beginning of the study, 34 subjects (19 female, 12 male, age: $22 \pm 3$ years, body height: $173 \pm 7 \mathrm{~cm}$, body mass: $66 \pm 9 \mathrm{~kg}$ ) volunteered to participate in this study after documenting their informed consent. Three subjects dropped out from the study due to time constraints, so consequently only 31 participants completed the study. All subjects were novice golfers and were told not to inform themselves about golfing during the study. The study was approved by the ethics committee of the University of Konstanz and conducted in accordance with the Declaration of Helsinki.

\subsection{Experimental design}

In this intervention study the subjects were assigned to one of three groups: the first and second group (EA, $n=10$; LA, $n=11$ ) had to practice a golf swing (a pitch), whereas the control group (CON, $n=10$ ) did not practice. Golf swing training was executed using an endpoint controlled golf robot (see Fig. 1). The movement trajectory of the robot guided golf swing was identical for both intervention groups except for the wrist angle progression during the backswing: the EA (early angle) group prac ticed the robot guided golf pitch with an early rotation of the club handle, resulting in an early radial abduction of the wrist during the backswing phase, whereas LA (late angle) group practiced the golf pitch with a late rotation of the club handle, resulting in a late radial abduction of the wrist during the backswing phase. Note that using only one training group might have been insufficient, as it could have been possible that early or late bending is already the natural behavior in novice golfers, thus impeding any training effects. The participants were neither told about the existence of two training groups nor about the slight difference in the movement pattern. Instead, they were told to focus on the starting position, swing velocity and the club position at the end of the backswing. To assess the changes due to the robotic guided training, a set of six free golf pitches was recorded one day before the training period (PRE), two days after the last training session (POST), and finally seven days after the last training session during the retention test (follow up, FUP; see Fig. 2).

\subsection{Apparatus}

In this study, we used a special robotic system (TopSwing, Sofea Software GmbH, Berlin, Germany), which operates as an end effector. A golf club handle is fixed to the robotic system. Connected to six computer controlled motor driven axes, this golf club handle can guide movement trajectories of different golf swings with adjustable velocity. Both the starting position and the movement trajectory can be adjusted to the individual anthropometrics of the subject. The trajectories of the three almost identical golf pitches (neutral, EA and LA) were programmed by a professional golf coach. They only 


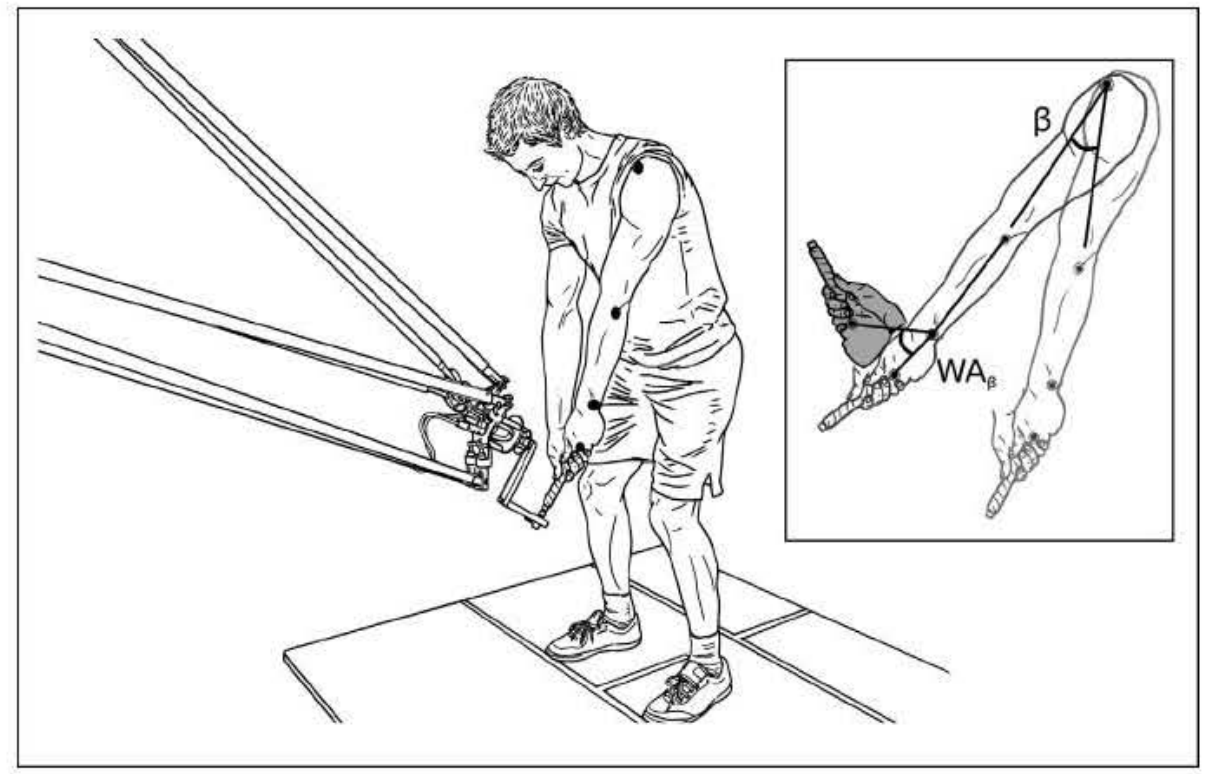

Fig. 1. Subject practicing the golf pitch using the endpoint-controlled golf robot (TopSwing, Sofea Software GmbH, Berlin, Germany). The markers for the motion capturing system were placed at the second metacarpus bone, radial styloid process, caput radii, and humeral greater tubercle of the left arm. On the right, an illustration of the wrist angle $W A_{\beta}$ and the arm angle $\beta$.

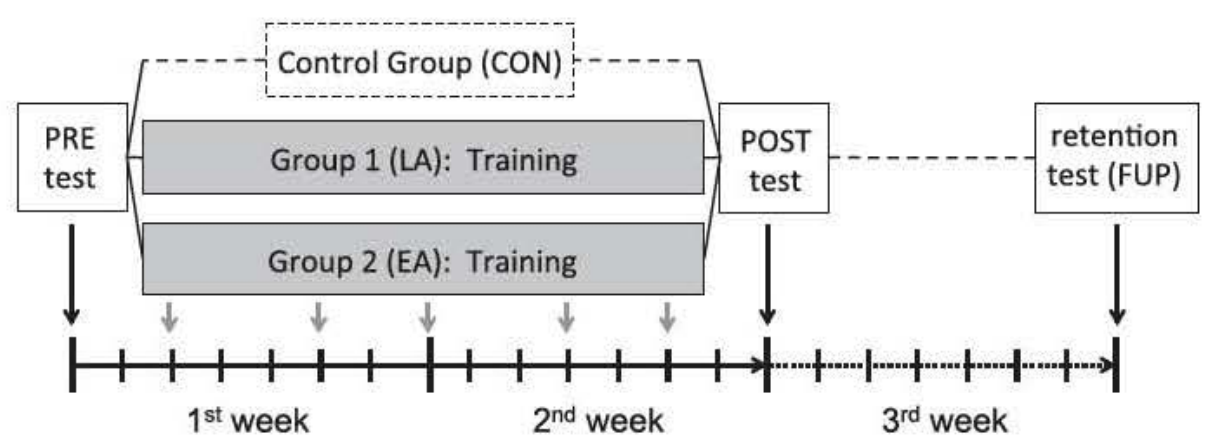

Fig. 2. Schematic illustration of the study design. After the PRE test ( 6 free golf swings), the subjects were assigned to one of the three groups (CON, EA, \& LA), matched with respect to wrist angle progression. All subjects of the EA and LA group practiced five times in the two weeks between PRE and POST test, whereas the control group (CON) had no training. After an additional week without training, the retention test (FUP) was performed.

differed with respect to the rotation of the golf club handle i.e., wrist bending during the back swing phase. The wrist angle during backswing was chosen as it has been shown that it plays a major role for both accuracy and distance of a golf shot (Chu, Sell, \& Lephart, 2010; Nesbit, 2005). One of the programmed pitches featured an early rotating club handle, one a late rotating club handle, and the third had a slight and continuously rotating club handle over the whole backswing phase. However, all the other parameters of the three trajectories (starting position, top position, end position, velocity, and overall trajectory except for the starting time of the club rotation) were identical. The start posi tion (height and lie angle of the golf club handle) of the robotic system was adjusted to each individual participant's start position. Then, the scale factor of the movement trajectory was adjusted during 3 golf swings using the neutral robot trajectory. To ensure a constant starting position during training 
and testing, the subject's foot position was marked on the floor and the subject was always instructed to (1) place their feet on the marks on the floor, knees slightly bent, (2) grasp the golf club handle of the robotic system with an interlocking grip, (3) slightly pronate the left hand when grasping the handle, and (4) fully extend the left elbow joint.

\subsection{Kinematic recordings}

During each of the three test sessions, six free golf pitches were recorded with a motion analysis system (LUKOtronic, Austria). Sampling frequency of the active marker system was set to $100 \mathrm{~Hz}$. Four markers were placed on the following anatomical landmarks of the left arm: second metacarpus bone, radial styloid process, caput radii, and humeral greater tubercle. The marker positions were marked on the skin before removal to facilitate correct marker placement during the POST and FUP test. These four markers were used to calculate the 3D angle of the left $\operatorname{arm}(\beta$; between radial styloid process, humeral greater tubercle and the plumb line) and the $3 \mathrm{D}$ angle of the left wrist $\left(W A_{\beta}\right.$; between second metacarpus, radial styloid process, and caput radii, see Fig. 1). Furthermore, we calculated the mean velocity of the arm during the backswing phase.

\subsection{Matching procedure}

To minimize mean group differences with respect to the pre training wrist angle progression, the three groups were matched after the PRE test. This was done by calculating the angle between the left arm and the plumb line at the time when the wrist angle first reached $20^{\circ}$ during backswing. Accord ing to the mean of this angle for their six golf swings, subjects were then assigned to one of the three groups, generating homogenous groups with respect to the mean angle between arm and plumb line.

\subsection{PRE, POST \& FUP tests}

To ensure consistent conditions, the subjects were reminded of the correct top position (end of the backswing) prior to each of the three tests as follows: the robot was set to the individual top position, where the subject grasped the shaft of the golf club while standing on the foot markers. Then, the sub ject stepped back from the robot and reproduced this top position with a normal golf club, using the correct top position of the robot golf club in front of him as a reference. This procedure was repeated three times before each test. Afterwards, the subject was again reminded of the correct foot place ment, interlocking grip with slight pronation and the full extension of the left elbow joint before each of the six free golf swings. In addition, the subject was instructed to focus on the top position of the backswing and to reproduce the velocity of the golf robot (which was set to a club head velocity of $0.4 \mathrm{~m} / \mathrm{s}$ during backswing and $0.8 \mathrm{~m} / \mathrm{s}$ during downswing). If their swing velocity was too fast, they were instructed to go slower during the next swing.

\subsection{Training}

During the two weeks of training, the subjects of group EA and LA had five training sessions with the robotic golf system. One training session consisted of 5 bouts of 10 robot guided golf pitches followed by 2 normal golf pitches, i.e., a total of 60 golf swings per session and a grand total of 300 golf swings, 250 of them robot guided. During guidance, the subjects were prompted to actively fol low the motion of the robot. There was always at least one day of rest between two training sessions. The control group did not receive any training. Before each training session, the subject was reminded of the correct starting position and was instructed to reproduce the top position of the backswing and the velocity of the robot during their free swings.

\subsection{Data processing}

The wrist angle $\left(W A_{\beta}\right)$ normalized to the wrist angle at the beginning of the movement position was calculated for seven arm positions during backswing to determine differences in the wrist angle 
between the groups. The seven arm positions also normalized to the initial arm position= $0^{\circ}$ were $=5^{\circ}, 10^{\circ}, 15^{\circ}, 20^{\circ}, 25^{\circ}$, and $30^{\circ}$ (see Fig. 1).

To detect the phases with the most pronounced changes in wrist angle during backswing $\left(030^{\circ}\right.$ arm position), we calculated the stepwise wrist angle change $\left(\Delta W A_{\beta}\right)$ between the seven $5^{\circ}$ incre ments during the backswing by the following formula:

$\Delta W A_{\beta} \quad \Delta W A_{\beta} 5 \quad W A_{\beta}$

For each measurement (PRE, POST and FUP), $W A_{\beta}$ as well as $\Delta W A_{\beta}$ were averaged over the six free swings that were performed without guidance. In addition, robot guided swings were recorded during the third and last training sessions to determine the wrist angle progression of the guided trajectories $\left(W A_{\beta}(\right.$ robot $\left.)\right)$ as a reference.

The backswing velocity $\left(V_{\text {back }}\right)$ was calculated via the marker on the radial styloid process and pro vided data about the velocity even during non guided golf swings. The average backswing velocity was calculated as the mean of $V_{-}$[back\} from $5^{\circ}$ to $30^{\circ}$ of the backswing.

\subsection{Statistics}

One way analyses of variance (ANOVA) with repeated measures were used to detect the effect of the robot guided training on the wrist angle progression $W A_{\beta}$ during the backswing and group differ ences in the backswing velocity $V_{\text {back }}$. The group affiliation [EA, LA, CON] was the between subjects factor, and the time points [PRE, POST, FUP] were repeated measures.

In addition, group differences in wrist angle change $\Delta W A_{\beta}$ during the backswing were analyzed with a mixed design ANOVA with repeated measures. The arm position $\beta\left[5^{\circ}, 10^{\circ}, 15^{\circ}, 20^{\circ}, 25^{\circ}\right.$, $30^{\circ}$ ] was the within subjects factor, the group affiliation [EA, LA, CON] was the between subjects factor, and the time points [PRE, POST, FUP] were repeated measures.

To ascertain differences in the wrist angle progression between the two different guided trajecto ries, $W A_{\beta}$ (robot) was tested with a mixed model ANOVA using the between subjects factor group [EA, LA] and the within subject factor arm position $\beta\left[5^{\circ}, 10^{\circ}, 15^{\circ}, 20^{\circ}, 25^{\circ}, 30^{\circ}\right]$.

In the case of significant differences $(p<.05)$, post hoc analyses were carried out and corrected for multiple comparisons (Bonferroni). Group data are presented as mean \pm standard deviation (SD).

\section{Results}

The ANOVA of the normalized wrist angle $\left(W A_{\beta}\right)$ showed a significant time $\times$ group effect. Post hoc analyses revealed a significant increase in EA's wrist angle during the backswing from PRE to POST $(p<.001$; see Fig. 3). In contrast, no significant changes could be observed in LA and CON. This

CON

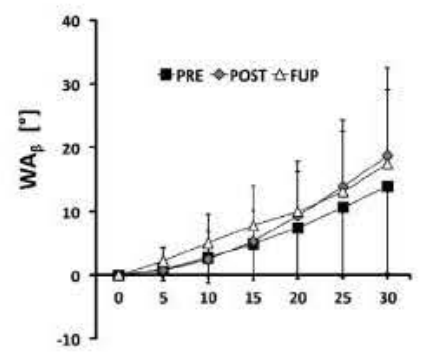

EA

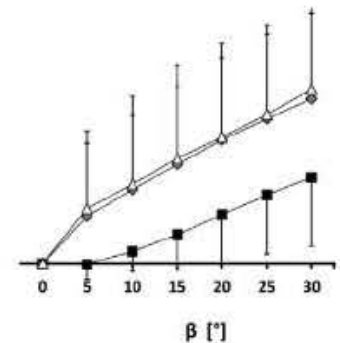

LA

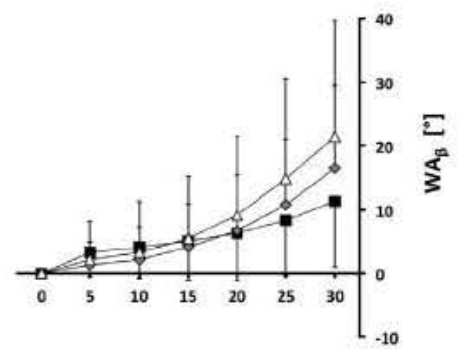

Fig. 3. Wrist angle progression (mean $\pm S D$ ) during the three tests - before (PRE), after (POST), and one week after (FUP) the training - in the control group (CON, left), the early angle group (EA, middle) and the late angle group (LA, right). In the EA group, the wrist angle decreased from PRE to POST in every recorded arm position between $5^{\circ}$ and $30^{\circ}$, and this change persisted during FUP. In LA and CON, no statistically significant changes over time could be observed, although changes in the later positions are visible for $L A$. 


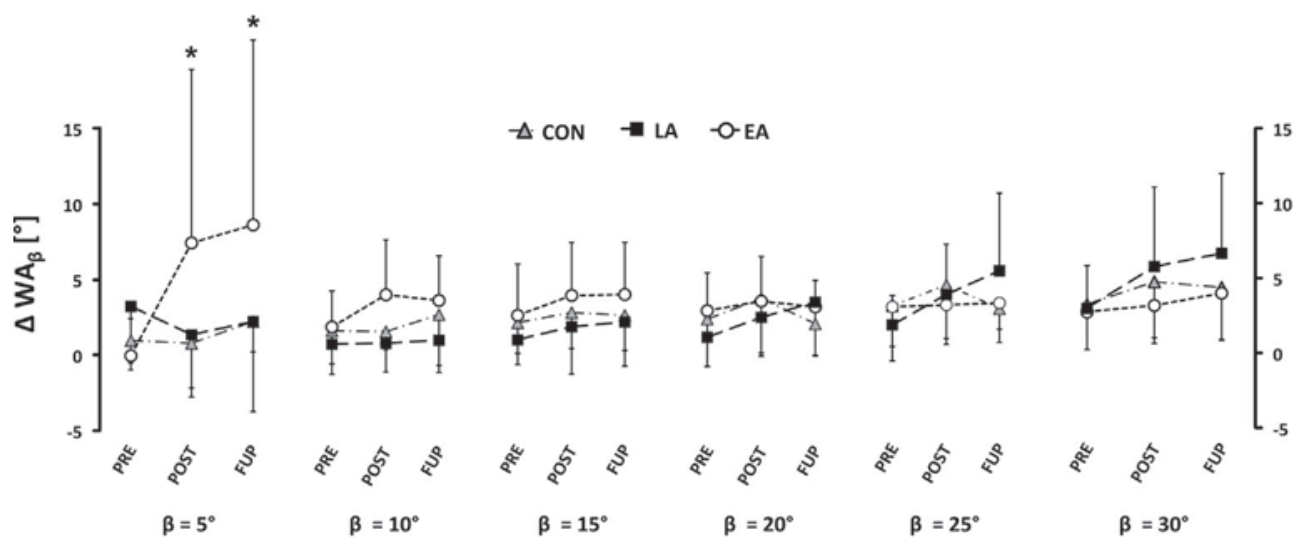

Fig. 4. The wrist angle changes (mean $\pm \mathrm{SD}$ ) in steps of $5^{\circ}$, i.e., in the first of the six parts of the Fig. (left, $5^{\circ}$ ), the changes in wrist angle from the $0^{\circ}$ starting position to the $5^{\circ}$ arm angle are depicted; in the second one $\left(10^{\circ}\right)$, the changes in wrist angle from the $5^{\circ} \mathrm{arm}$ angle to the $10^{\circ} \mathrm{arm}$ angle, and so on. EA's wrist angle changes are most pronounced during the first $5^{\circ}$ (statistically significant increase from PRE to POST, persisting during FUP; ${ }^{*} p<.001$ ). LA shows some changes - although statistically not significant - during the later arm angles, whereas there are no changes in CON.

significant increase in EA persisted until the retention test 7 days after the last training $(p<.001)$. Again, no significant changes in the mean wrist angle appeared in LA and CON during the retention test.

The mixed design ANOVA of $\Delta W A_{\beta}$ showed a significant time $\times$ group $\times$ arm angle interaction effect: after practicing the golf swing, the wrist angle change increased in the EA group, whereas LA's $\Delta W A_{\beta}$ decreased after the training and no changes occurred in CON. Post hoc analyses showed that this interaction was due to the first $5^{\circ}$ of the arm angle during backswing $(p<.001)$. In EA, $\Delta W A_{5^{\circ}}$ significantly increased by $7^{\circ}$ from PRE to POST, whereas $\Delta W A_{5^{\circ}}$ of LA decreased slightly by $2^{\circ}$ (see Fig. 4). In CON, $\Delta W A_{5^{\circ}}$ did not change. Those changes persisted until FUP: $\Delta W A_{5^{\circ}}$ even increased further by $2^{\circ}$ in EA and $1^{\circ}$ in LA and CON.

Before the training intervention, i.e., during PRE measurements, no significant differences were present in $W A_{\beta}$ between EA, LA, and CON. The backswing velocity $V_{\text {back }}$ did not differ between groups either, and there was no significant group $\times$ time interaction effect.

As expected, the wrist angle progression during the robot guided training $\left(W A_{\beta}(\right.$ robot $\left.)\right)$ differed significantly between EA and LA $(p<.05)$. Post hoc analyses showed a significantly larger wrist angle $\left(W A_{\beta}(\right.$ robot $\left.)\right)$ for EA compared to LA during every arm position of the backswing (see Fig. 5).

\section{Discussion}

The main finding of the present study is that the robotic guidance training was successful in shap ing the intended movement pattern of a sport specific motor task, in this case the wrist angle progres sion during a golf pitch. In addition, this effect was not temporary, but was still present in the retention test one week after the end of the training.

The findings of the present study expand on the results of Feygin et al. (2002) and Liu et al. (2006), who showed that haptic guidance can be used to shape isolated arm movements in a sitting position. In the present study, we were able to demonstrate that this is also possible for the movement pattern of a sport specific whole body movement with many degrees of freedom. This is an important step for the application of haptic guidance in sports and motor rehabilitation, as learners usually do not strug gle with isolated movements, but with whole body movements that require a precise coordination of multiple body parts usually an interdependent kinematic chain in space and time.

One reason why complex whole body movements like these are not easy to learn is that they impose high task dependent information processing demands on the athlete (Wulf \& Shea, 2002), 


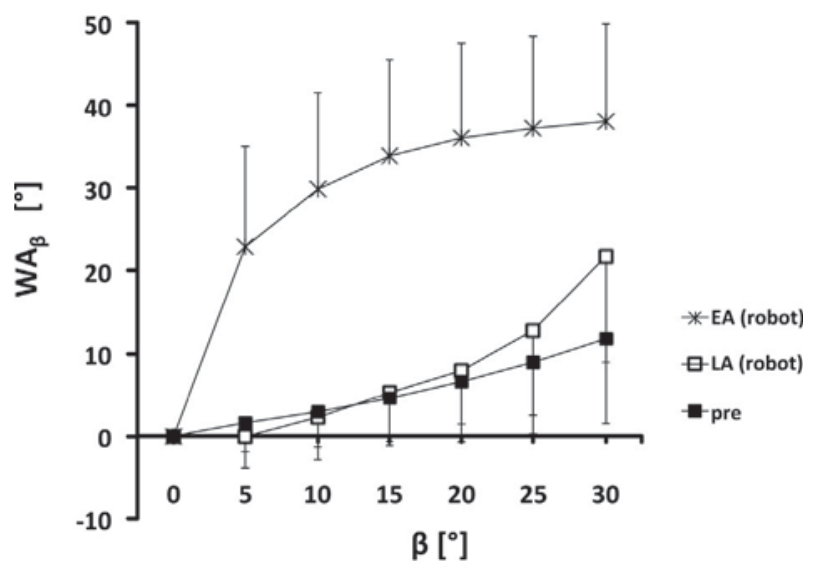

Fig. 5. Wrist angle progression during the robot-guided golf swings (mean $\pm \mathrm{SD}$ ) for all participants of the early angle (EA) group and the late angle (LA) group. For comparison's sake, the grand mean of all participants in the PRE test is also shown. The difference between the naive movement trajectory before the training (PRE) and the guided trajectory that the EA group practiced is bigger than the difference between the PRE and LA trajectories. This disparity stems from the fact that the two robot-guided trajectories were defined before the PRE test.

which can be overwhelming for novices. Haptic guidance does not require the learner to focus on all the aspects of the correct movement; instead, he just has to follow the correct movement. Therefore, it is very likely that haptic guidance greatly reduces the information processing demand, thus enabling novices to learn a complex whole body movement without having to pay attention to the various key points of the movement. Our results support this hypothesis, as the participants' attention was direc ted towards the starting position, the swing velocity and the club position at the end of the backswing, whereas the wrist angle progression was never mentioned to the subjects. It can be argued that with holding declarative knowledge or instructions about the target task reduces or even prevents hypoth esis testing and can thus be regarded as an implicit motor learning situation (Masters, 1992; Zhu, Poolton, \& Masters, 2012). Implicit motor learning has been shown to result in more robust perfor mance compared to explicit motor learning, especially under psychological stressful conditions (Mullen, Hardy, \& Oldham, 2007) or when multitasking (for review see Zhu et al., 2012). There is also evidence that haptic guidance can improve performance while freeing attention for a secondary task (Griffiths \& Gillespie, 2005). From this perspective, haptic guidance might have similar effects as implicit motor learning, as it might reduce information processing demands and thus minimize the participation of the working memory during acquisition. To pinpoint the mechanisms involved in robot guided motor learning and investigate similarities to implicit learning, future studies should assess the verbal knowledge the participants gathered about the task.

Another interesting aspect of robotic guidance that warrants further investigation is the frequency of this haptic feedback: in the present study, the subjects in the training groups always performed two non guided swings after every ten robot guided swings. The guidance hypothesis states that the lear ner can develop a dependency on the augmented feedback he receives during acquisition (haptic guid ance in this case), resulting in a decreased performance when feedback is removed during a retention or transfer test (Schmidt, 1991). Winstein, Pohl, and Lewthwaite (1994) provided some evidence for the guidance hypothesis. They demonstrated that a high dosage of augmented feedback (especially physical guidance) has detrimental effects on the learning of a positioning movement. In contrast, studies investigating the effect of physical guidance or assistance on the learning of a complex motor task (such as balancing tasks or oscillatory movements) revealed that guidance can facilitate the motor learning process (Wulf, Shea, \& Matschiner, 1998; Wulf et al., 1998). For complex motor tasks it has been postulated that a reduction of information processing demands in a complex movement enables the performer to first acquire a movement representation. It has been suggested that this is especially applicable for novices without any perception about the motor task (Sigrist et al., 2013; 
Wulf \& Shea, 2002). This is in line with the challenge point theory, which states that a motor task might not be learned if the acquisition is too challenging (Guadagnoli \& Lee, 2004). For novices, a high amount of feedback in the case of robotic guidance a high number of guided repetitions and a low number of non guided repetitions may be most suitable, whereas for more advanced learners, the number of non guided repetitions should probably be increased, consistent with the idea of fading feedback, i.e., a reduction of feedback frequency with increasing task mastery (Crowell \& Davis, 2011; Kovacs \& Shea, 2011). Still, the question remains open whether $100 \%$ feedback frequency for novices would be even more helpful. In the case of robotic guidance, this would translate to training without any interspersed non guided trials. The notion of motor skill learning as the formation of an internal movement model of the desired movement pattern (Dingwell, Mah, \& Mussa Ivaldi, 2002), by an error based learning process of the motor system (Emken \& Reinkensmeyer, 2005; Reinkensmeyer \& Patton, 2009) would suggest that at least some interspersed non guided trials are necessary. Otherwise, no comparison between the guided trials and the free trials would be possible, and this comparison i.e., the difference between the intended and the actual movement, in other words, the error has been suggested to be one of the most common mechanisms underlying motor learning (Wolpert, Diedrichsen, \& Flanagan, 2011).

The results of the present study provide evidence that haptic guidance with a few interspersed tri als was definitely successful in teaching a movement. However, we cannot say whether there is an optimal relationship between guided and non guided trials to facilitate the learning of a movement pattern.

In addition to the feedback frequency, another open question in the context of robotic guidance is the degree of active participation during the guided trials. There are few studies on this subject, but their results suggest that passively following the guided movement may not be optimal: for example, Beets et al. (2012) provided evidence that passive guidance improved spatiotemporal performance of a bimanual coordination pattern, but that active training of the motor task was much more effective compared to passive guidance. Lotze, Braun, Birbaumer, Anders, and Cohen (2003) showed that pas sively guiding a subject's limb through a movement was followed by reduced cortical excitability and less activation of the primary motor cortex. In contrast, active motor training is supposed to lead to the encoding of a motor memory on the primary motor cortex, expressed by an enhanced corticomotor excitability of agonist muscles and depressed excitability of antagonist muscles (Kaelin Lang, Sawaki, \& Cohen, 2005). In the present study, the participants were instructed to actively follow the motion of the robot guided golf club. By visual inspection of the participants while training and their feedback we are convinced that they were active throughout the movement, however, the subjects' degree of active participation was not directly measured. Future studies could address the question whether, and how much, different degrees of participation (e.g. amount and direction of the applied force) affect the learning of a motor task.

In addition to facilitating motor learning in healthy subjects, robotic guidance with interspersed free trials might also be helpful for patients trying to re learn a movement during neurorehabilitation, as it has been suggested that active participation is important for the success of robotic guidance in neurorehabilitaion (Hesse, Schmidt, Werner, \& Bardeleben, 2003; Lum, Burgar, Shor, Majmundar, \& Van der Loos, 2002) and that repeated practice of a particular movement is crucial for motor recovery (Bütefisch, Hummelsheim, Denzler, \& Mauritz, 1995).

When comparing the effect of the robotic guidance intervention on the two training groups EA and LA, it is apparent that EA showed significant changes in the wrist angle progression, while LA only showed a slightly increased wrist angle in the later phases of the backswing ( $25^{\circ}$ and $30^{\circ}$ arm angle, see Fig. 4). As the matching procedure ensured similar wrist angle progressions between the groups, the lack of changes in LA's wrist angle progression can most likely be explained by the small differ ences between the initial wrist angle progression before the training and the wrist angle progression the LA group was subjected to during the guided trials (see Fig. 5). In contrast to LA, the difference between EA's robot guided and the initial movement trajectory was sufficient to induce significant changes in the movement pattern.

Although this study only aimed to shape a movement pattern by means of haptic guidance, it would be interesting to see how a robot improved movement pattern translates to improvements in performance. For a golf swing, it has been shown that the wrist angle progression plays a major role 
for both accuracy and distance (Chu et al., 2010; Nesbit, 2005). Therefore, it is very likely that the changes we have observed after robotic guidance directly affect the performance in a golf shot.

\section{Conclusion}

In summary, the results of the present study showed that haptic guidance in combination with interspersed free trials were able to shape the movement pattern of a novel complex sport specific motor task, and that these changes persisted after seven days without any further training.

Although further research is needed to refine training parameters such as the ratio between robot guided trials and free trials, or the amount of active participation during robot guided trials, we conclude that endpoint controlled robotic guidance has a lot of potential in facilitating the motor learning of novel complex sport specific movements by enabling the learner to experience the correct movement right from the start.

\section{Acknowledgments}

The authors want to thank Sascha Orlic from TopSwing Golfschule Berlin for his help with the adjustment of the robotic device and programming the golf pitch trajectories, the company Sofea $\mathrm{GmbH}$, Berlin for providing the robotic device for the duration of this study and Lucie Pfeiffer for her help with the data acquisition and training supervision.

\section{Appendix A}

Formulae and equations

Eq. (1): Calculation of the relative changes of the wrist angle between the subsequent arm positions.

\section{References}

Beets, I. A. M., Macé, M., Meesen, R. L. J., Cuypers, K., Levin, O., \& Swinnen, S. P. (2012). Active versus passive training of a complex bimanual task: Is prescriptive proprioceptive information sufficient for inducing motor learning? PloS One, 7(5), e37687.

Bütefisch, C., Hummelsheim, H., Denzler, P., \& Mauritz, K.-H. (1995). Repetitive training of isolated movements improves the outcome of motor rehabilitation of the centrally paretic hand. Journal of the Neurological Sciences, 130(1), 59-68.

Chu, Y., Sell, T. C., \& Lephart, S. M. (2010). The relationship between biomechanical variables and driving performance during the golf swing. Journal of Sports and Sciences, 28(11), 1251-1259.

Crowell, H. P., \& Davis, I. S. (2011). Gait retraining to reduce lower extremity loading in runners. Clinical biomechanics, 26(1), 78-83.

Dingwell, J. B., Mah, C. D., \& Mussa-Ivaldi, F. A. (2002). Manipulating objects with internal degrees of freedom: Evidence for model-based control. Journal of Neurophysiology, 88(1), 222-235.

Emken, J. L., \& Reinkensmeyer, D. J. (2005). Robot-enhanced motor learning: accelerating internal model formation during locomotion by transient dynamic amplification. IEEE Transactions on Neural Systems and Rehabilitation Engineering, 13(1), 33-39.

Feygin, D., Keehner, M., \& Tendick, R. (2002). Haptic guidance: Experimental evaluation of a haptic training method for a perceptual motor skill. Paper presented at the 10 th international symposium on haptic interfaces for virtual environment and teleoperator systems (Haptics, 2002).

Griffiths, P. G., \& Gillespie, R. B. (2005). Sharing control between humans and automation using haptic interface: Primary and secondary task performance benefits. Human Factors: The Journal of the Human Factors and Ergonomics Society, 47(3), $574-590$.

Guadagnoli, M. A., \& Lee, T. D. (2004). Challenge point: A framework for conceptualizing the effects of various practice conditions in motor learning. Journal of Motor Behavior, 36(2), 212-224.

Hesse, S., Schmidt, H., Werner, C., \& Bardeleben, A. (2003). Upper and lower extremity robotic devices for rehabilitation and for studying motor control. Current Opinion in Neurology, 16(6), 705-710.

Huang, V. S., \& Krakauer, J. W. (2009). Robotic neurorehabilitation: A computational motor learning perspective. Journal of NeuroEngineering and Rehabilitation, 6(1), 5.

Kaelin-Lang, A., Sawaki, L., \& Cohen, L. G. (2005). Role of voluntary drive in encoding an elementary motor memory. Journal of Neurophysiology, 93(2), 1099-1103.

Kovacs, A. J., \& Shea, C. H. (2011). The learning of 90 continuous relative phases with and without Lissajous feedback: External and internally generated bimanual coordination. Acta Psychologica, 136(3), 311-320. 
Kwakkel, G., Kollen, B. J., \& Krebs, H. I. (2008). Effects of robot-assisted therapy on upper limb recovery after stroke: A systematic review. Neurorehabilitation and Neural Repair, 22(2), 111-121.

Liu, J., Cramer, S. C., \& Reinkensmeyer, D. J. (2006). Learning to perform a new movement with robotic assistance: Comparison of haptic guidance and visual demonstration. Journal of NeuroEngineering and Rehabilitation, 3, 20.

Lotze, M., Braun, C., Birbaumer, N., Anders, S., \& Cohen, L. G. (2003). Motor learning elicited by voluntary drive. Brain, 126(4), 866-872.

Lum, P. S., Burgar, C. G., Shor, P. C., Majmundar, M., \& Van der Loos, M. (2002). Robot-assisted movement training compared with conventional therapy techniques for the rehabilitation of upper-limb motor function after stroke. Archives of Physical Medicine and Rehabilitation, 83(7), 952-959.

Masters, R. S. W. (1992). Knowledge, knerves and know-how: The role of explicit versus implicit knowledge in the breakdown of a complex motor skill under pressure. British Journal of Psychology, 83(3), 343-358.

Mullen, R., Hardy, L., \& Oldham, A. (2007). Implicit and explicit control of motor actions: revisiting some early evidence. British Journal of Psychology, 98(1), 141-156.

Nesbit, S. M. (2005). A three dimensional kinematic and kinetic study of the golf swing. Journal of Sports Science and Medicine, 4(4), 499-519.

Newell, K. M. (1991). Motor skill acquisition. Annual Review of Psychology, 42(1), 213-237.

Norouzi-Gheidari, N., Archambault, P. S., \& Fung, J. (2012). Effects of robot-assisted therapy on stroke rehabilitation in upper limbs: Systematic review and meta-analysis of the literature. Journal of Rehabilitation Research and Development, 49(4), 479-496.

Prange, G. B., Jannink, M. J. A., Groothuis-Oudshoorn, C. G. M., Hermens, H. J., \& Ijzerman, M. J. (2006). Systematic review of the effect of robot-aided therapy on recovery of the hemiparetic arm after stroke. Journal of Rehabilitation Research and Development, 43(2), 171.

Reinkensmeyer, D. J., \& Patton, J. L. (2009). Can robots help the learning of skilled actions? Exercise and Sport Sciences Reviews, $37(1), 43$.

Sanger, T. D. (2004). Failure of motor learning for large initial errors. Neural Computation, 16(9), 1873-1886.

Schmidt, R. A. (1991). Frequent augmented feedback can degrade learning: Evidence and interpretations. Tutorials in Motor Neuroscience, 62, 59-75.

Sigrist, R., Rauter, G., Riener, R., \& Wolf, P. (2013). Augmented visual, auditory, haptic, and multimodal feedback in motor learning: A review. Psychonomic Bulletin E Review, 20(1), 21-53.

Sivan, M., O'Connor, R. J., Makower, S., Levesley, M., \& Bhakta, B. (2011). Systematic review of outcome measures used in the evaluation of robot-assisted upper limb exercise in stroke. Journal of Rehabilitation Medicine, 43(3), 181-189.

Winstein, C. J., Pohl, P. S., \& Lewthwaite, R. (1994). Effects of physical guidance and knowledge of results on motor learning: Support for the guidance hypothesis. Research Quarterly for Exercise and Sport, 65(4), 316-323.

Wolpert, D. M., Diedrichsen, J., \& Flanagan, J. R. (2011). Principles of sensorimotor learning. Nature Reviews Neuroscience, 12(12), 739-751.

Wulf, G., \& Shea, C. H. (2002). Principles derived from the study of simple skills do not generalize to complex skill learning. Psychonomic Bulletin E Review, 9(2), 185-211.

Wulf, G., Shea, C. H., \& Matschiner, S. (1998). Frequent feedback enhances complex motor skill learning. Journal of Motor Behavior, 30(2), 180-192.

Wulf, G., Shea, C. H., \& Whitacre, C. A. (1998). Physical-guidance benefits in learning a complex motor skill. Journal of Motor Behavior, 30(4), 367-380

Zhu, F., Poolton, J. M., \& Masters, R. S. W. (2012). Neuroscientific aspects of implicit motor learning in sport. In W. T. Albert Gollhofer \& J. B. Nielsen (Eds.), Handbook of motor control and motor learning (pp. 155). Abington: Routledge International. 